University of Wollongong

Research Online

Australian Institute for Innovative Materials -

Papers

Australian Institute for Innovative Materials

$1-1-2019$

Three-Dimensional Porous Cobalt Phosphide Nanocubes Encapsulated in a Graphene Aerogel as an Advanced Anode with High Coulombic Efficiency for High-Energy Lithium-Ion Batteries

Hong Gao

University of Wollongong, hg173@uowmail.edu.au

Fuhua Yang

University of Wollongong, fy896@uowmail.edu.au

Yang Zheng

University of Wollongong, yz966@uowmail.edu.au

Qing Zhang

University of Wollongong, qz964@uowmail.edu.au

Junnan Hao

University of Wollongong, jh845@uowmail.edu.au

See next page for additional authors

Follow this and additional works at: https://ro.uow.edu.au/aiimpapers

Part of the Engineering Commons, and the Physical Sciences and Mathematics Commons

Research Online is the open access institutional repository for the University of Wollongong. For further information contact the UOW Library: research-pubs@uow.edu.au 


\title{
Three-Dimensional Porous Cobalt Phosphide Nanocubes Encapsulated in a Graphene Aerogel as an Advanced Anode with High Coulombic Efficiency for High-Energy Lithium-lon Batteries
}

\author{
Abstract \\ An ingeniously designed porous structure can synergistically optimize the desired properties and \\ maximize the advantages of a material as an electrode for a high-performance energy storage system. \\ The active material with a porous nanostructure could reduce the ion diffusion path and buffer the strain \\ caused by the volume changes during cycling. Furthermore, combining the active material with a three- \\ dimensional (3D) graphene aerogel (GA) matrix is an ideal way to maintain the structural integrity, \\ improve the conductivity, and overcome the aggregation problem of the nanomaterials. Herein, we \\ adopted a facile template-based strategy to derive a composite of 3D hierarchically porous cobalt \\ phosphide nanocubes with a graphene aerogel (CoP@GA). The as-prepared CoP@GA features porous \\ cobalt phosphide nanocubes that are firmly encapsulated and uniformly distributed in the well-defined \\ graphene aerogel skeleton. Benefiting from the hierarchical porosity, structural integrity, and conductive \\ network, the CoP@GA electrode manifests an ultrahigh initial Coulombic efficiency (88.6\%), outstanding \\ lithium storage performance in terms of excellent cycling performance ( $805.3 \mathrm{mAh} \cdot \mathrm{g}-1$ after 200 cycles at \\ $200 \mathrm{~mA} \cdot \mathrm{g}-1)$, superior high-energy performance (351.8 $\mathrm{mAh} \cdot \mathrm{g}-1$ after 4000 cycles at $10 \mathrm{~A} \cdot \mathrm{g}-1)$, and \\ exceptional rate capability. Moreover, this synthesis protocol could be an instructive precedent for \\ fabricating transition-metal-phosphide-based 3D porous composites with excellent electrochemical \\ performances. \\ Disciplines \\ Engineering | Physical Sciences and Mathematics

\section{Publication Details} \\ Gao, H., Yang, F., Zheng, Y., Zhang, Q., Hao, J., Zhang, S., Zheng, H., Chen, J., Liu, H. \& Guo, Z. (2019). Three- \\ Dimensional Porous Cobalt Phosphide Nanocubes Encapsulated in a Graphene Aerogel as an Advanced \\ Anode with High Coulombic Efficiency for High-Energy Lithium-Ion Batteries. ACS Applied Materials and \\ Interfaces, 11 (5), 5373-5379.
}

\section{Authors}

Hong Gao, Fuhua Yang, Yang Zheng, Qing Zhang, Junnan Hao, Shilin Zhang, Hao Zheng, Jun Chen, HuaKun Liu, and Zaiping Guo 


\title{
Three-Dimensional Porous Cobalt Phosphide Nanocubes Encapsulated in a Graphene Aerogel as an Advanced Anode with High Coulombic Efficiency for High-Energy Lithium-Ion Batteries
}

\author{
Hong Gao, ${ }^{\dagger}$ Fuhua Yang, ${ }^{\dagger}$ Yang Zheng, ${ }^{\dagger}$ Qing Zhang, ${ }^{\dagger}$ Junnan Hao, ${ }^{\dagger}$ Shilin Zhang, ${ }^{\oplus}$ Hao Zheng, ${ }^{\dagger}$ \\ Jun Chen, ${ }^{*}$, Huakun Liu, ${ }^{\dagger}$ and Zaiping Guo* $†$
}

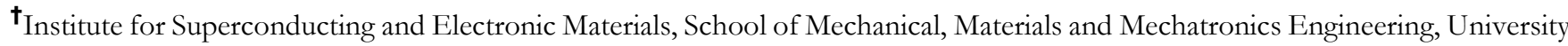
of Wollongong, North Wollongong, New South Wales 2500, Australia

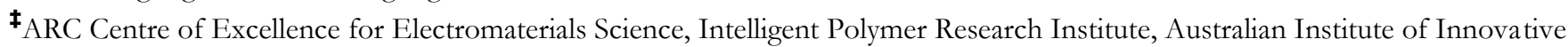
Materials, University of Wollongong, North Wollongong, New South Wales 2522, Australia

* Supporting Information

ABSTRACT: An ingeniously designed porous structure can synergistically optimize the desired properties and maximize the advantages of a material as an electrode for a high-performance energy storage system. The active material with a porous nanostructure could reduce the ion diffusion path and buffer the strain caused by the volume changes during cycling. Furthermore, combining the active material with a three-dimensional (3D) graphene aerogel (GA) matrix is an ideal way to maintain the structural integrity, improve the conductivity, and overcome the aggregation problem of the nanomaterials. Herein, we adopted a facile template-based strategy to derive a composite of 3D hierarchically porous cobalt phosphide nanocubes with a graphene aerogel (CoP@GA). The as-prepared CoP@GA features porous cobalt phosphide nanocubes that are firmly encapsulated and uniformly distributed in the well-defined graphene aerogel skeleton. Benefiting from the hierarchical porosity, structural integrity, and conductive network, the CoP@GA electrode manifests an

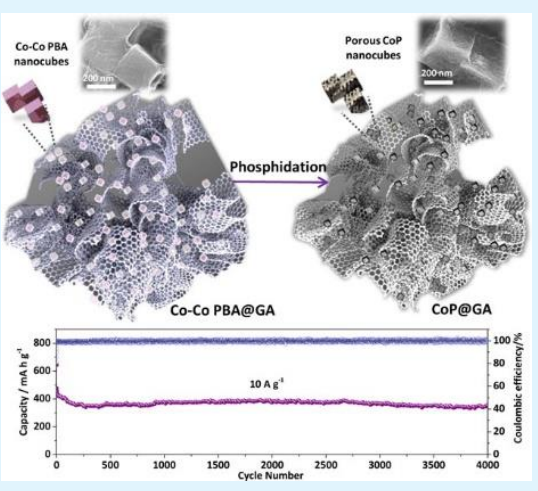
ultrahigh initial Coulombic efficiency (88.6\%), outstanding lithium storage performance in terms of excellent cycling performance $\left(805.3 \mathrm{mAh}^{\prime} \mathrm{g}^{-1}\right.$ after 200 cycles at $\left.200 \mathrm{~mA}^{*} \mathrm{~g}^{-1}\right)$, superior high-energy performance $\left(351.8 \mathrm{mAh}^{\circ} \mathrm{g}^{-1}\right.$ after 4000 cycles at $\left.10 \mathrm{~A}^{\cdot} \mathrm{g}^{-1}\right)$, and exceptional rate capability. Moreover, this synthesis protocol could be an instructive precedent for fabricating transition-metal-phosphide-based 3D porous composites with excellent electrochemical performances.

KEYWORDS: cobalt phosphide, graphene aerogel, template-engaged, stability, lithium-ion battery

\section{INTRODUCTION}

Lithium-ion batteries (LIBs) with superior rate performance as well as ultralong cycle life are currently in great demand to satisfy the ever-growing requirements of the consumer electronic market. ${ }^{1-8}$ Among the various anode materials, phosphorus has attracted much attention because of its superhigh theoretical specific capacity (2596 $\left.\mathrm{mAh}^{\prime} \mathrm{g}^{-1}\right)$. Due to its low conductivity, however, combining phosphorus with a conductive metal to obtain the metal phosphide is an ideal method to improve the conductivity of the whole electrode. ${ }^{9-18}$ In addition, the presence of conductive metals could buffer the volume expansion of phosphorus during repeated charge-discharge processes. What is more, the conversion-type reaction mechanism of the metal phosphide makes it able to react reversibly with larger amounts of $\mathrm{Li}$ ions per formula unit than is possible with the conventional intercalation-type mechanism, leading to much higher lithium storage capacity than that of graphite-based anode materials. ${ }^{12}$

Unfortunately, the metal phosphides still face the challenges of low initial Coulombic efficiency, short cycle life, and poor high-rate capability derived from the poor electric conductivity and volume variation during the $\mathrm{Li}^{+}$intercalation/deintercalation process. ${ }^{19-24}$ The ingeniously designed porous structure can synergistically optimize the desired properties and maximize the advantages of the active material. The active material with a porous nanostructure could buffer the strain caused by the volume changes during the cycling process, and the multiple interior voids could reduce the ion diffusion paths and facilitate the immersion of electrolyte in electrode materials. ${ }^{25-32}$ Nevertheless, it is widely recognized that such nanomaterials are vulnerable to agglomeration during synthesis and electrochemical processes. ${ }^{33}$ Therefore, encapsulation of the nanomaterials in a three-dimensional (3D) conductive matrix to prevent the aggregation is quite necessary. The graphene aerogel (GA), with a novel $3 \mathrm{D}$ porous graphene architecture, has ample active sites, good mechanical flexibility, and an interconnected 3D conductive network; therefore, it is believed to be a promising electrode configuration for promoting electron and ion transfer,

Received: November 18, 2018

Accepted: January 11,2019

Published: January 11, 2019 


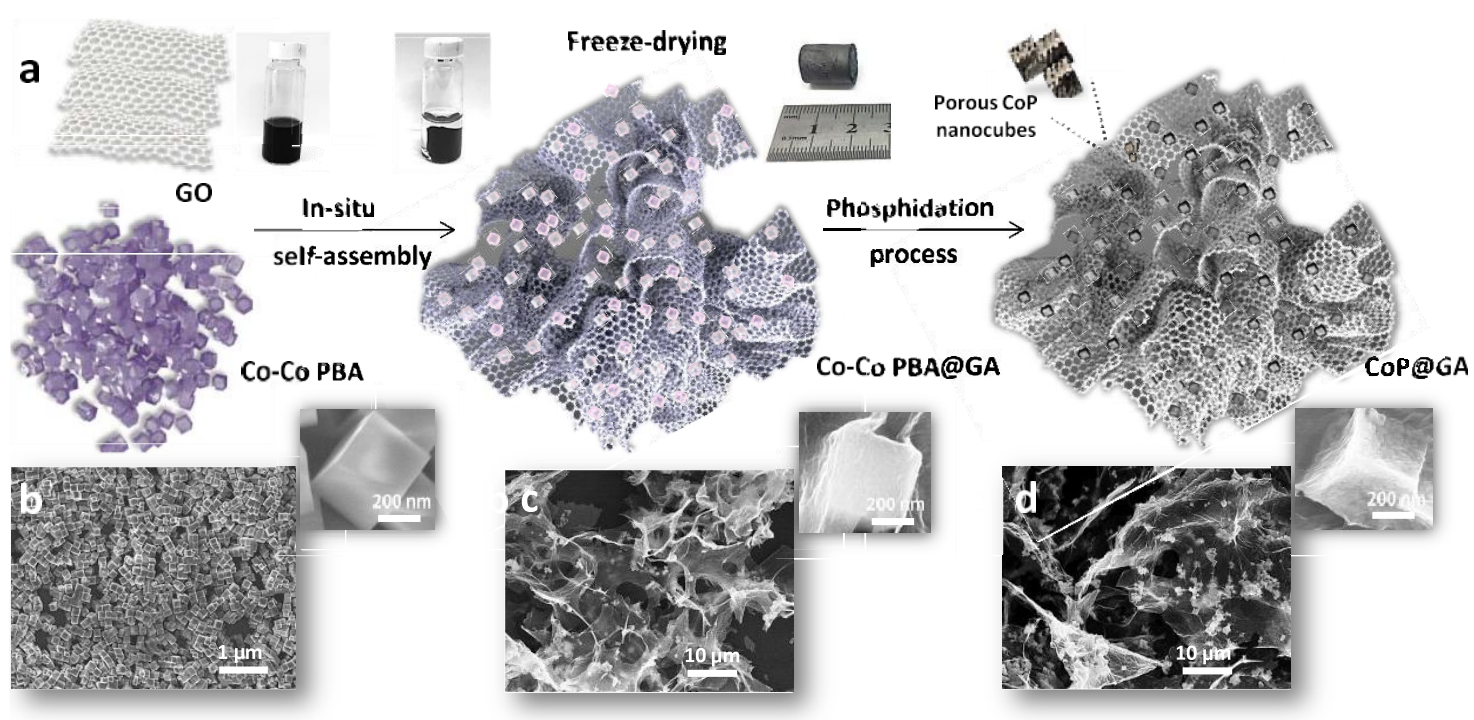

Figure 1. (a) Schematic illustration of the fabrication process for the CoP@GA composite. Field-emission scanning electron microscopy (FESEM) images of (b) Co-Co PBA, (c) Co-Co-PBA@GA, and (d) CoP@GA.

maintaining the structural integrity, and improving the Coulombic efficiency and cycling stability of the electrode during cycling. ${ }^{34,35}$

Of the various metal phosphides, cobalt phosphide (CoP), with a high theoretical capacity $\left(894 \mathrm{mAh}^{\prime} \mathrm{g}^{-1}\right.$ ) and relatively low charge and discharge voltage platforms, is favorable as an anode material. ${ }^{15,36,37}$ Nevertheless, CoP features the same volume variation and relative low conductivity issues as other metal phosphides, as mentioned above. Herein, we adopted the Co-Co Prussian blue analogue (PBA) as the template combined with the GA through a low-temperature gas phase phosphidation process to get the CoP@GA composite. PBA, which belongs to the class of metal-organic frameworks, has various morphologies that are controllable, of a uniform size, and have distinctive reactivity and thermal behavior, which make it an ideal self-sacrificial precursor to derive porous nanostructured materials. ${ }^{38-40}$ The uniquely designed CoP@GA framework features porous CoP nanocubes that are uniformly distributed and firmly encapsulated in the 3D graphene aerogel construction. The merits of the CoP@GA composite are listed as follows: (i) the porous CoP nanocubes are wrapped by the GA to accommodate the volume change of $\mathrm{CoP}$ and ensure structural integrity during the cycling process; (ii) the GA offers a $3 \mathrm{D}$ conductive network to facilitate electron transfer and enable the whole framework immersed in the electrolyte sufficiently. All of these appropriate advantages are integrated into the CoP@GA composite, and excellent lithium storage properties could be anticipated. The as-prepared CoP@GA shows an initial discharge capacity of $1212.9 \mathrm{mAh}^{*} \mathrm{~g}^{-1}$ with initial Coulombic efficiency of $88.6 \%$, delivering a capacity of 805.3 $\mathrm{mAh} \cdot \mathrm{g}^{-1}$ during the 200th cycle at the current density of 200 $\mathrm{mA}^{*} \mathrm{~g}^{-1}$. At a high current density of $10 \mathrm{~A}^{*} \mathrm{~g}^{-1}$, the CoP@GA electrode still exhibits a capacity of $351.8 \mathrm{mAh}^{\circ} \mathrm{g}^{-1}$ even after 4000 cycles.

\section{RESULTS ANDDISCUSSION}

Figure 1 a exhibits the facile fabrication process for the CoP@GA composite. The preparation process includes three steps: (i) a precipitation method to produce the highly uniform $\mathrm{CO}_{3}\left(\mathrm{Co}_{-}\right.$ $\left.(\mathrm{CN})_{6}\right)_{2}$ Prussian blue analogue nanocubes (Figure 1b), denoted $\mathrm{Co}-\mathrm{Co} \mathrm{PBA}$; (ii) homogeneous in situ encapsulation of Co-Co PBA nanocubes in the graphene aerogel, followed by a freeze-drying process to obtain the 3D porous $\mathrm{Co}-\mathrm{Co} \mathrm{PBA} @$ GA precursor (Figure 1c); and, subsequently, (iii) a thermally induced phosphidation process to obtain the final product, CoP@GA composite, which still maintains the integrated 3D porous framework (Figure 1d). Simultaneously, the graphene aerogel is subjected to a further thermal-induced reduction. The as-prepared CoP@GA features porous CoP nanocubes homogeneously distributed/encapsulated in the 3D GA framework.

Figure 2a presents a field-emission scanning electron microscopy (FESEM) image of Co-Co PBA nanocubes with an average size of $\sim 300 \mathrm{~nm}$. After the phosphidation process, the pristine CoP well retains the cubic shape of the Co-Co PBA precursor with a little change in size (Figures $2 \mathrm{~b}$ and S4a,b, Supporting Information). An intriguing feature of $\mathrm{CoP}$ is that the internal cubic structure appears porous with a crosslike interior. This is because the cobalt ions are liberated from $\mathrm{Co}-$ Co PBA to form CoP during the thermal annealing process (Figure $\mathrm{S} 4 \mathrm{c}-\mathrm{e}$ ). The $\mathrm{N}_{2}$ adsorption-desorption isotherm of the pristine CoP is shown in Figure S5. The calculated specific surface area of CoP is $69.53 \mathrm{~m}^{2 \cdot} \mathrm{g}^{-1}$ with the pore volume calculated to be $\sim 0.165 \mathrm{~cm}^{3 \cdot} \mathrm{g}^{-1}$ with most of the pores $\sim 2.01$ $\mathrm{nm}$ in size, which confirms the porous nature of the pristine CoP as well. The elemental mapping images of CoP demonstrate the presence of cobalt, phosphorus, and carbon elements (Figure S4f-i). Figure 2c shows a FESEM image of the Co-Co PBA@ GA precursor, in which the solid Co-Co PBA nanocubes with smooth surfaces are encapsulated in the graphene aerogel framework. After the healing process, the CoP nanocubes in the CoP@GA composite remain wrapped by the graphene aerogel and the structure of CoP is still similar to that of the porous pristine CoP (Figure 2d). The bright- and dark-field transmission electron microscopy (TEM) images of CoP@GA show that the interior structure of CoP in the CoP@GA composite is identical to that of the pristine CoP (Figure 2e,f). The electron diffraction pattern of CoP@GA in the inset of Figure 2e can be identified as resulting from the (011), (211), (013), (201), and (020) planes of CoP. The corresponding elemental mapping images demonstrate that the cobalt, phosphorus, and carbon 

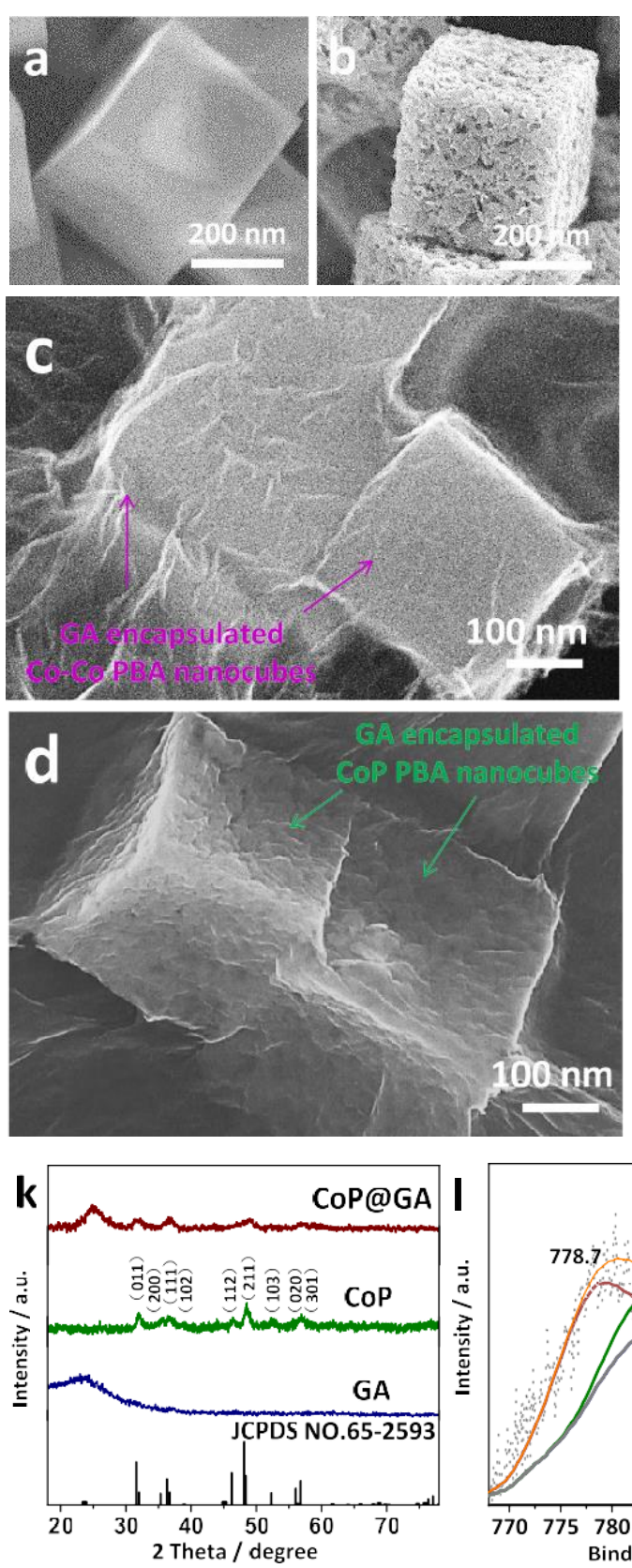
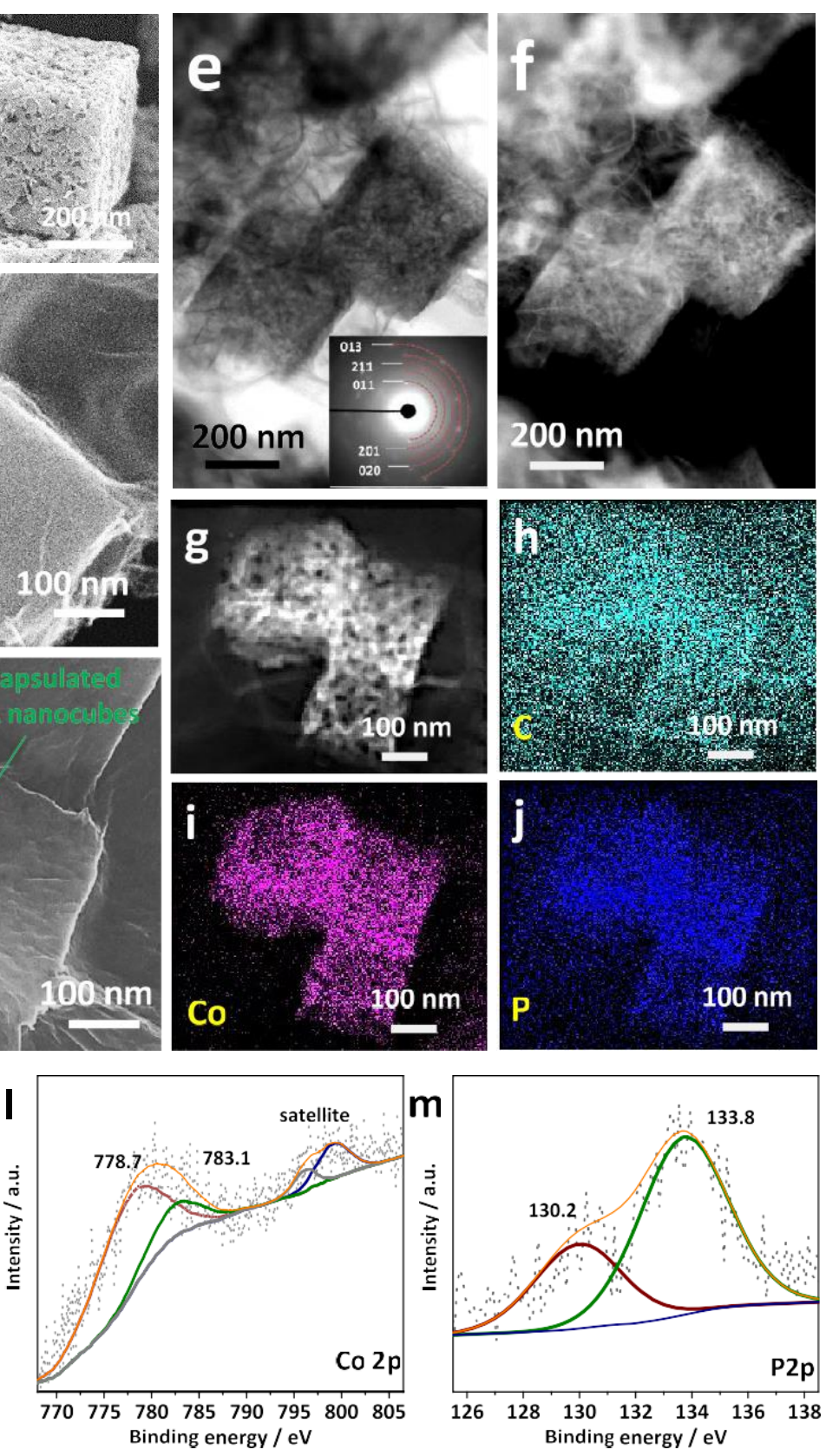

Figure2.SEMimages of (a)Co-CoPBA precursor, (b) pristineCoP, (c)Co-CoPBA@GA, and(d)CoP@GA composite. (e)Bright-and(f)darkfield images of CoP@GA; the inset of (e) shows the corresponding electron diffraction pattern. (g) Scanning TEM image and element mapping images of (h) carbon, (i) cobalt, and (j) phosphorus. (k) X-ray diffraction (XRD) patterns of CoP@GA, CoP, and GA. X-ray photoelectron spectroscopy (XPS) analysis of CoP@GA: (l) Co 2p and (m) P 2p.

elements are all uniformly distributed throughout the nanocube (Figure $2 \mathrm{~g}-\mathrm{j}$ ).

The X-ray diffraction (XRD) pattern in Figure 2k indicates that the Co-Co PBA precursor has been successfully converted to pristine CoP and CoP@GA. The CoP@GA also exhibits the distinctive XRD peaks of GA, which feature a hump at 22$28^{\circ} .{ }^{41}$ The XRD pattern of the Co-Co PBA precursor is shown in Figure S6, in which all of the diffraction peaks can be ascribed to cubic $\mathrm{Co}_{3}\left[\mathrm{Co}(\mathrm{CN})_{6}\right]_{2}$ (JCPDS no. 77-1161). The X-ray photoelectron spectroscopy (XPS) survey spectrum of the CoP@GA composite in Figure S7 demonstrates the presence of $\mathrm{Co}, \mathrm{P}$, and $\mathrm{C}$ elements. The high-resolution XPS spectra of the Co and P elements in CoP@GA are shown in Figure 21,m, respectively. The peaks at $778.7 \mathrm{eV}$ in the $\mathrm{Co} 2 \mathrm{p}$ region and
$130.2 \mathrm{eV}$ in the $\mathrm{P} 2 \mathrm{p}$ region are assigned to the binding energies of $\mathrm{Co}$ and $\mathrm{P}$ in $\mathrm{CoP}$, whereas the peaks at $783.1 \mathrm{eV}$ in the Co $2 \mathrm{p}$ region and $133.8 \mathrm{eV}$ in the $\mathrm{P} 2 \mathrm{p}$ region are close to those for the oxidized $\mathrm{Co}$ and $\mathrm{P}$ species, which are derived from the superficial oxidation of the materials. ${ }^{42}$ The peaks between 794 and $805 \mathrm{eV}$ in the Co $2 \mathrm{p}$ region are ascribed to the satellite signal. ${ }^{43}$ Figure S8 shows the Raman spectra of CoP@GA and graphene oxide (GO). One of the two obvious peaks at around $1344 \mathrm{~cm}^{-1}$ in both the CoP@GA and GO curves corresponds to a defective carbon band (D band), whereas the other peaks at around 1589 $\mathrm{cm}^{-1}$ correspond to the graphitic carbon band (G band). ${ }^{44}$ The D/G intensity ratio of CoP@GA (1.24) is higher than that of pure GO (1.08), demonstrating that $\mathrm{GO}$ was reduced to 

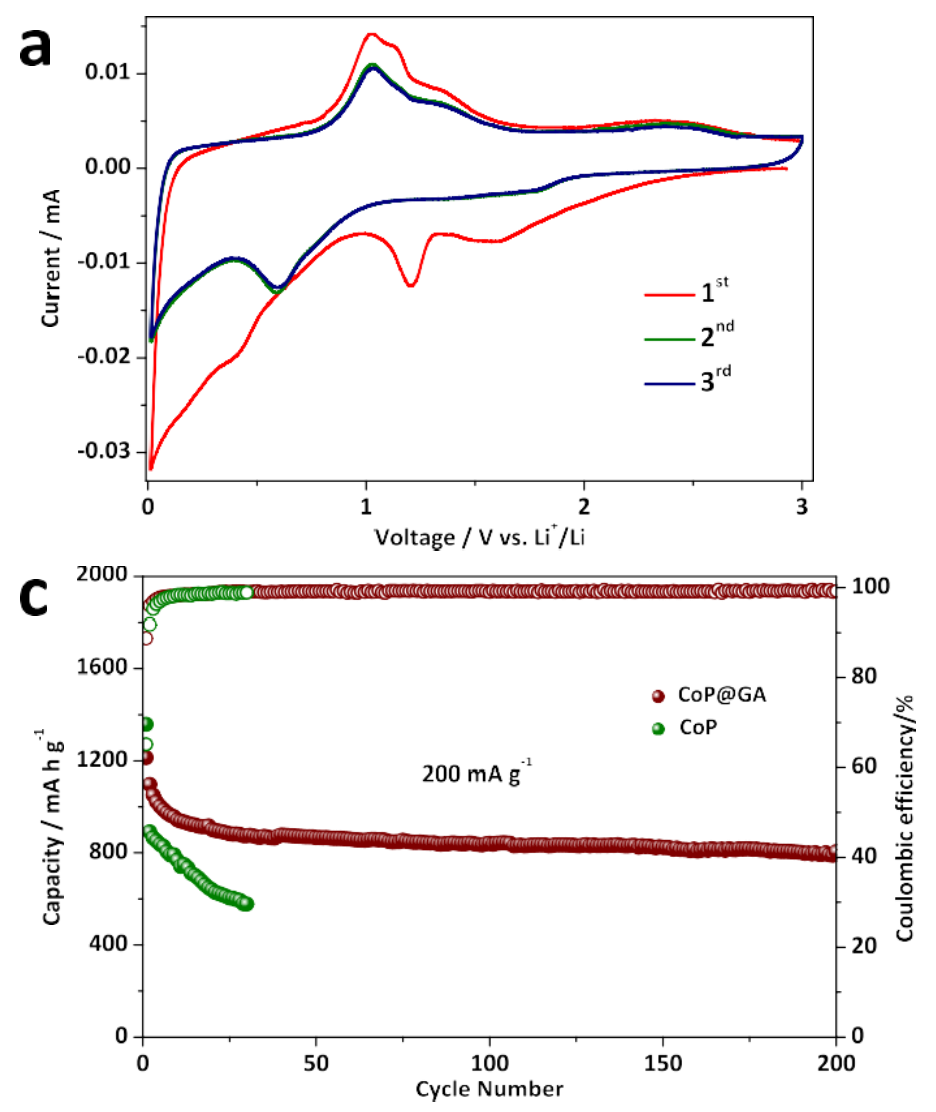
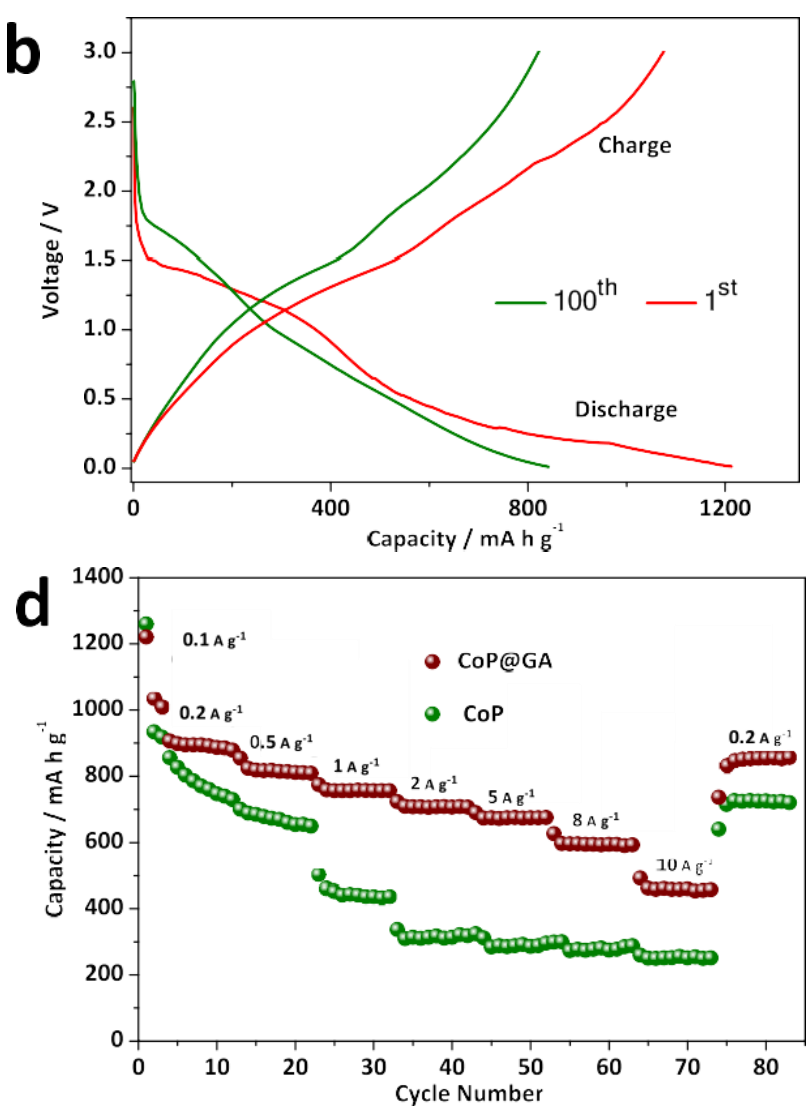

Figure 3. Electrochemical performance of the CoP@GA hybrid for LIBs: (a) cyclic voltammograms of the first three cycles of CoP@GA; (b) chargedischarge voltage profiles of the CoP@GA electrode at a current density of $200 \mathrm{~mA} \mathrm{~g}^{-1}$; comparison of (c) cycling and (d) rate capacities of CoP@GA and CoP electrodes.

graphene in CoP@GA. The graphene content for CoP@GA is estimated via the thermogravimetric analysis (Figure S9).

CoP@GA was investigated as an anode for LIBs. Figure 3a exhibits the cyclic voltammogram (CV) curves obtained between 0.01 and $3 \mathrm{~V}$ at a scan rate of $0.1 \mathrm{mV}^{*} \mathrm{~s}^{-1}$. A peak appearing at $\sim 1.2 \mathrm{~V}$ in the initial cathodic scan most likely corresponds to the conversion reaction $\mathrm{CoP}+3 \mathrm{Li}^{+}+3 \mathrm{e}^{-} \rightarrow \mathrm{Co}$ $+\mathrm{Li}_{3} \mathrm{P}$. A following weak peak at $\sim 0.48 \mathrm{~V}$ was detected, which corresponds to the reaction $\mathrm{CoP}+\mathrm{Li}^{+}+\mathrm{e}^{-} \rightarrow \mathrm{Co}+\mathrm{LiP}$ and the formation of the solid electrolyte interphase (SEI) layer. A major peak at $\sim 1.1 \mathrm{~V}$ and a minor peak at $\sim 2.4 \mathrm{~V}$ are observed in the initial anodic scan, which belong to the decomposition of the solid electrolyte interphase layer and the reaction $\mathrm{Li}_{3} \mathrm{P} \rightarrow \mathrm{LiP}+$ $2 \mathrm{Li}^{+}+2 \mathrm{e}^{-}$, respectively. The CV curves are in agreement with the reported results. ${ }^{20,45}$ After the first scan, the remaining curves manifest identical cathodic and anodic peaks, indicating good reversibility and high Coulombic efficiency. It is worth mentioning that the redox peaks of CoP@GA in CV curves well match the potential plateaus in the charge-discharge lines (Figure $3 \mathrm{~b}$ ). The charge-discharge curves of the prinstine CoP electrode are exhibited in Figure S10.

Figure $3 c$ displays the cycling performance of CoP@GA and CoP electrodes at a current density of $200 \mathrm{~mA}^{*} \mathrm{~g}^{-1}$, within a voltage range of $0.01-3 \mathrm{~V}$ for LIBs. The current densities and specific capacities in this work are all calculated on the basis of the mass of the active material, except for binder and carbon black. Obviously, the CoP@GA electrode offers much higher capacity and more stable cycling performance than those of the pristine CoP. The first discharge capacity for the CoP@GA electrode was $1212.9 \mathrm{mAh}^{*} \mathrm{~g}^{-1}$, and the corresponding initial
Coulombic efficiency of $88.6 \%$ was achieved. Compared to that of the previously reported cobalt phosphide as an anode for LIBs, our work shows an excellent initial Coulombic efficiency (Table S1). After 200 cycles, a capacity of $805.3 \mathrm{mAh}^{\circ} \mathrm{g}^{-1}$ can be obtained. The initial capacity for the CoP@GA electrode is higher than the theoretical specific capacity for CoP $(894 \mathrm{mAh}$. $\left.\mathrm{g}^{-1}\right)$. This is mainly due to the formation of the SEI layer during the first discharge process. ${ }^{46-49}$ Compared to that of the CoP@ GA electrode, the discharge capacity of the pristine CoP electrode dramatically fades to $577.2 \mathrm{mAh}^{\circ} \mathrm{g}^{-1}$ after only 30 cycles. At $2 \mathrm{~A}^{\prime} \mathrm{g}^{-1}$, the CoP@GA electrode maintains a stable cycling life and a discharge capacity of $667.7 \mathrm{mAh}^{\prime} \mathrm{g}^{-1}$ can be observed over 200 cycles (Figure S11). Compared with the previously reported counterparts, our work shows superior cycling performance (Table S2). The cycling performance of the pristine GA for LIBs is shown in Figure S12. The rate capabilities of both CoP@GA and CoP were evaluated at the current densities of $0.1,0.2,0.5,1,2,5,8$, and $10 \mathrm{~A}^{\prime} \mathrm{g}^{-1}$ (Figure 3d). The CoP@GA electrode clearly shows much better rate capability than that of the CoP electrode. The specific capacities are 1032.2, 895, 818.1, 756, 707.4, 674.4, 595.5, and 459.2 $\mathrm{mA}^{\circ} \mathrm{g}^{-1}$ at current densities ranging from 0.1 to $10 \mathrm{~A}^{*} \mathrm{~g}^{-1}$. When the current density is reversed back to $0.2 \mathrm{~A}^{\circ} \mathrm{g}^{-1}$, the specific capacity of $853.8 \mathrm{mAh}^{\circ} \mathrm{g}^{-1}$ can still be obtained. The sodium storage properties of the CoP@GA electrode were studied as well (Figure S13).

Benefiting from the unique framework, the CoP@GA electrode shows a superior lithium storage capacity even at the high current density of $10 \mathrm{~A}^{\prime} \mathrm{g}^{-1}$. It delivers a discharge capacity of $351.8 \mathrm{mAh}^{\circ} \mathrm{g}^{-1}$ after 4000 cycles (Figure 4a). Figure S14 

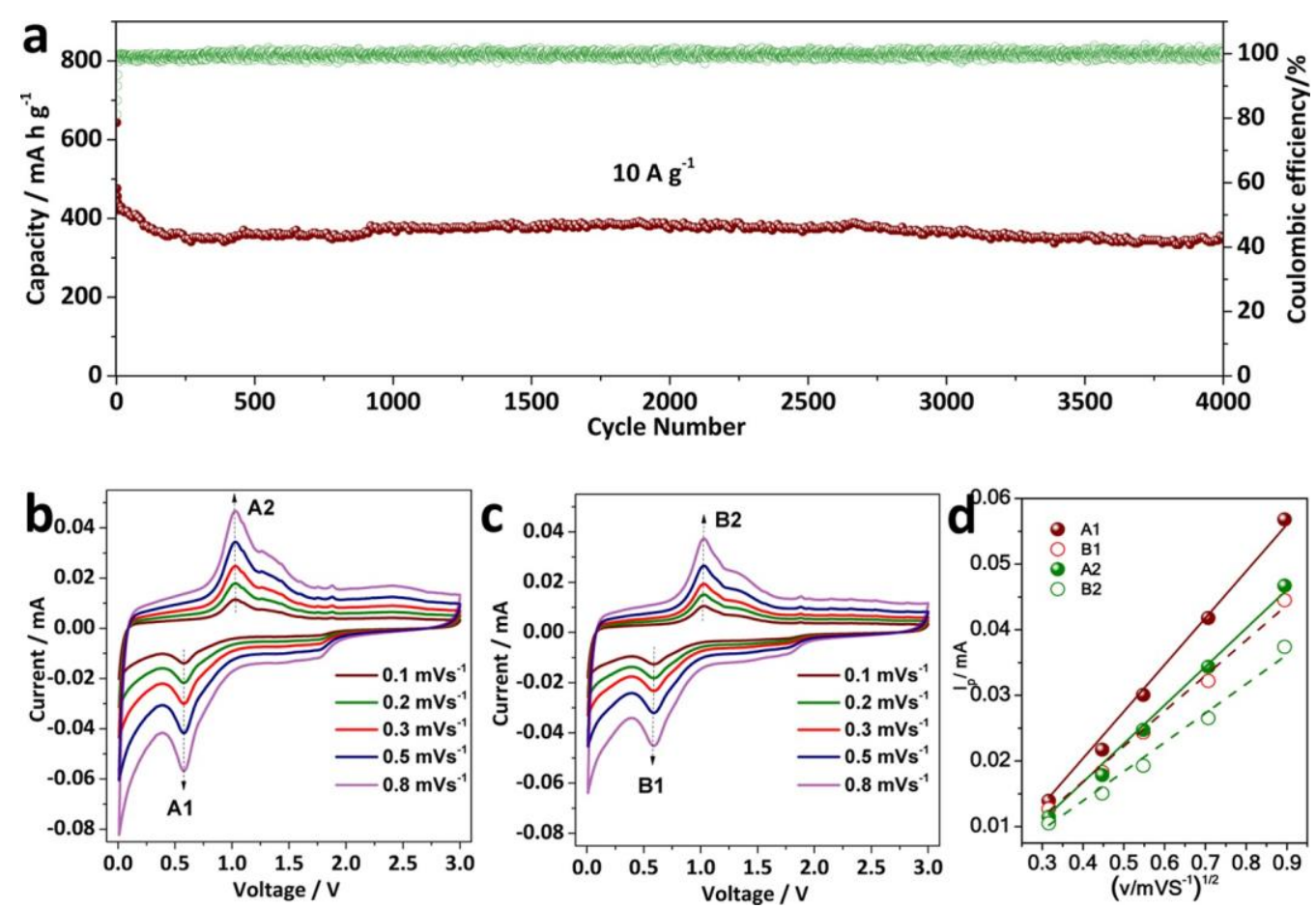

Figure 4. (a) Long cycling performance of the CoP@GA electrode at the high current density of $10 \mathrm{~mA}^{\cdot} \mathrm{g}^{-1}$. (b) CV curves of CoP@GA and (c) CoP electrode at different scan rates. (d) Linear relationship of the peak currents for the peaks labeled in (b) and (c) versus the square root of the scan rate $\left[\mathrm{V}^{0.5} \mathrm{~s}^{-0.5}\right]$ and the related linear fits.

presents FESEM and bright- and dark-field TEM images of the CoP@GA electrode before and after 200 cycles. Aftercycling, the $3 \mathrm{D}$ structure is still maintained; the encapsulated CoP cubic structure is preserved, demonstrating the excellent structural stability of the electrode; and the existence of the GA guarantees the structural integrity and improves the cycling stability. In addition, GA offers a highly conductive 3D network to ensure $\mathrm{rapid} /$ efficient electron transportation. It is widely recognized that the electrochemical reaction rate is diffusion-controlled. Herein, we have recorded CV curves for both CoP@GA and $\mathrm{CoP}$ at different scan rates ranging from 0.1 to $0.8 \mathrm{mV}^{*} \mathrm{~s}^{-1}$ (Figure $4 b, c)$. The intensities of both cathodic and anodic peaks in the $\mathrm{CV}$ curves change with the scan rate. Based on the Randles-Sevcik equation, ${ }^{30}$ a linear relationship can be obtained between the peak current $\left(I_{\mathrm{p}}\right)$ and the scan rate $(\mathrm{V})$, as presented in Figure $4 \mathrm{~d}$. It is obvious that $I_{\mathrm{p}}$ shows a linear relationship with v ${ }^{1 / 2}$ and the CoP@GA electrode exhibits a higher diffusion coefficient than that of the pristine CoP, corresponding to the previous rate performance. The electrochemical impedance spectroscopy results for the CoP@GA and CoP electrodes after 50 cycles further demonstrate the high conductivity of the CoP@GA electrode. The charge transfer resistance of CoP electrode is higher than that of the CoP@GA electrode over 50 cycles, demonstrating the fast charge transfer rate of the CoP@GA electrode (Figure S15).

\section{CONCLUSIONS}

A template-based synthesis protocol was developed to incorporate porous $\mathrm{CoP}$ nanocubes into the 3D GA matrix with a homogeneous distribution through a facile in situ encapsulation, followed by a thermally induced phosphidation process to obtain the CoP@GA hybrid. The porous CoP nanocubes are homogeneously distributed and firmly encapsulated in a well-defined porous GA skeleton, which provides a highly conductive 3D framework as well as offers effective accommodation to buffer the large volume collapse of CoP during the cycling process. In addition, the specially designed structure enables the construction to be immersed in the electrolyte sufficiently. Therefore, excellent electrochemical performance could be anticipated. The as-prepared CoP@GA electrode exhibits a capacity of $805.3 \mathrm{mAh}^{*} \mathrm{~g}^{-1}$ during the 200th cycle at $200 \mathrm{~mA}^{\prime} \mathrm{g}^{-1}$ and exhibits a capacity of $351.8 \mathrm{mAh}^{\circ} \mathrm{g}^{-1}$ even at $10 \mathrm{~A}^{\prime} \mathrm{g}^{-1}$ after 4000 cycles. Moreover, since the metal ions and organic ligands in the metal-organic frameworks can be adjusted easily, the proposed template-based approach is a facile and cost-effective synthesis protocol. It could be a precedent to guide the encapsulation of other metal-phosphide-based materials with large volume expansion properties.

\section{EXPERIMENTALSECTION}

4.1. Synthesis of CoP@GA Composites. 4.1.1. Preparation of Co-Co PBA. In a typical procedure, cobalt acetate $(0.15 \mathrm{~g})$ and sodium citrate $(0.27 \mathrm{~g})$ were dissolved in deionized water $(20 \mathrm{~mL})$ to form solution A. Potassium hexacyanocobaltate(III) (0.13 g) was also dissolved in deionized water $(20 \mathrm{~mL})$ to form solution B. After that, solutions $\mathrm{A}$ and $\mathrm{B}$ were mixed thoroughly under magnetic stirring. Then, the mixed solution was aged overnight at room temperature. Then, after centrifugation and washing with water and ethanol, the precipitates were collected and dried at $60^{\circ} \mathrm{C}$ overnight.

4.1.2. In Situ Self-Assembly of the Co-Co PBA with the Graphene Aerogel (Co-Co PBA@GA). Graphene oxide was fabricated by Hummers' method. For preparation of Co-Co PBA@GA, Co-Co $\mathrm{PBA}$ and GO in a weight ratio of $4: 1$ were added into $10 \mathrm{~mL}$ of deionized water in a vial, followed by vigorous stirring $(10 \mathrm{~min})$ and sonication (20 $\mathrm{min}$ ) and then hypophosphorous acid (HPA) was added (2-10 times the quantity of GO), followed by sonication for $10 \mathrm{~min}$. The function of HPA as a reductant in the in situ fabrication process is to promote the formation of the graphene hydrogel. Subsequently, the vial was placed in an oven at $60{ }^{\circ} \mathrm{C}$ overnight to obtain the $\mathrm{Co}-\mathrm{Co}$ PBA@GA precursor (Figure S1). Then, the precursor was washed with 
deionized water and freeze-dried under vacuum to develop Co-Co PBA@GA. The regularity of the network formation process for the 3D graphene hydrogel can be clearly observed, and the corresponding digital photograph illustrates that the volume of the graphene hydrogel varies with the mass of HPA (Figure S1f). It is worth noting that some of the $\mathrm{Co}-\mathrm{Co}$ PBA nanocubes are not encapsulated in the graphene hydrogel when the amount of HPA is 10 times the quantity of GO (Figure S1e). Considering the integrity and stability of the whole framework, the $\mathrm{Co}-\mathrm{Co} \mathrm{PBA} @ \mathrm{GA}$ precursor with an amount of HPA that is 8 times the quantity of GO was selected for further study.

4.1.3. Phosphidation Process to Obtain the Composite of Cobalt Phosphide with the Graphene Aerogel (CoP@GA).Co-Co PBA@GA and $\mathrm{NaH}_{2} \mathrm{PO}_{2}$ with 1:10 mole ratio of $\mathrm{Co}$ to $\mathrm{P}$ were put into a porcelain boat separately, and $\mathrm{NaH}_{2} \mathrm{PO}_{2}$ was placed in the upstream position in a tube furnace, followed by annealing the materials at $30{ }^{\circ} \mathrm{C}$ for $2 \mathrm{~h}$ (a heating rate of $3^{\circ} \mathrm{C} \cdot \mathrm{min}^{-1}$ ) in an Ar atmosphere with a flow rate of 50 sccm. After cooling under an Ar atmosphere, CoP@GA was obtained. For comparison, pristine $\mathrm{CoP}$ was fabricated under identical conditions using $\mathrm{Co}-\mathrm{Co} \mathrm{PBA}$ as the precursor. For comparison, calcination temperatures of 400,500 , and $600^{\circ} \mathrm{C}$ were also used to obtain the CoP400, CoP-500, and CoP-600 reference samples, respectively. The XRD pattern reveals that $\mathrm{CoP}-400$ should be identified with CoP (JCPDS no. 65-2593), the same as that for the CoP produced at $300^{\circ} \mathrm{C}$, whereas the CoP-500 and CoP-600 samples are composed of $\mathrm{Co}_{2} \mathrm{P}$ and $\mathrm{CoP}$ (Figure S2). The FESEM images of CoP-400, CoP-500, and CoP-600 show that the size of the nanocubes gradually becomes larger as the temperature increases (Figure S3).

4.2. Characterization. The crystalline structures of the samples were identified by X-ray diffraction (GBCMMA, XRD). Raman spectra were obtained on a JOBIN Yvon Horiba HR800 Raman spectrometer. $\mathrm{X}$-ray photoelectron spectroscopy was conducted on VG Multilab 2000. The morphologies and structures of the materials were studied using field-emission scanning electron microscopy (FESEM, JEOL JSM-7500FA) and transmission electron microscopy (TEM, JEOL 2011, $200 \mathrm{keV}$ ), respectively. The TEM was connected to an energydispersive spectral analysis system (probe-corrected JEOL ARM200F).

4.3. Electrochemical Characterizations. The electrochemical characterizations of the CoP@GA and CoP electrodes were conducted on CR2032 cointype cells. The electrodes were prepared through blending $80 \mathrm{wt} \%$ active materials with $10 \mathrm{wt} \%$ sodium carboxymethyl cellulose and $10 \mathrm{wt} \%$ Super P into distilled water. The obtained slurries were cast on a copper foil, dried in vacuum at $80^{\circ} \mathrm{C}$ overnight, and then pressed at $30 \mathrm{MPa}$. The mass loading of the active materials on individual electrodes was $1.5 \pm 0.2 \mathrm{mg}^{*} \mathrm{~cm}^{-2}$. Lithium metal was used as the reference electrode and counter electrode. A glass microfiber filter (Whatman) was used as the separator. $\mathrm{LiPF}_{6}\left(1.0 \mathrm{~mol}^{\circ} \mathrm{L}^{-1}\right)$ in an ethylene carbonate and diethyl carbonate solution $(1: 1 \mathrm{v} / \mathrm{v})$ was used as the electrolyte. A glove box under an Ar atmosphere was utilized to assemble the cell, which was tested by a Land battery testing system at room temperature at the voltage of $0.01-3.0 \mathrm{~V}$.

\section{AUTHOR INFORMATION}

\section{Corresponding Authors}

*E-mail: junc@uow.edu.au (J.C.).

*E-mail: zguo@uow.edu.au (Z.G.).

ORCID

Shilin Zhang: 0000-0002-3268-5708
Jun Chen: 0000-0003-2218-7382

Zaiping Guo: 0000-0003-3464-5301

Notes

The authors declare no competing financial interest.

\section{ACKNOWLEDGMENTS}

H.G. and F.Y. contributed equally to this work. Support from the Australian Research Council (ARC) through an ARC Discovery project (DP170102406), Future Fellowship (FT150100109), and ARC CoE Scheme (CE140100012) is gratefully acknowledged. The authors would also like to thank the Australian National Fabrication Facility (ANFF) for equipment access, the Electron Microscopy Centre (EMC) at the University of Wollongong for the electron microscopy characterizations, and Dr. T. Silver for critical reading of the manuscript.

\section{REFERENCES}

(1) Armand, M.; Tarascon, J.-M. Building Better Batteries. Nature 2008, 451, 652-657.

(2) Dunn, B.; Kamath, H.; Tarascon, J.-M. Electrical Energy Storage for the Grid: A Battery of Choices. Science 2011, 334, 928-935.

(3) Shen, X.; Liu, H.; Cheng, X.-B.; Yan, C.; Huang, J.-Q. Beyond Lithium Ion Batteries: Higher Energy Density Battery Systems Based on Lithium Metal Anodes. Energy Storage Mater. 2018, 12, 161-175.

(4) Guo, X.; Sun, B.; Su, D.; Liu, X.; Liu, H.; Wang, Y.; Wang, G. Recent Developments of Aprotic Lithium-Oxygen Batteries: Functional Materials Determine the Electrochemical Performance. Sci. Bull. 2017, 62, 442-452.

(5) Liu, Y.; Tai, Z.; Zhou, T.; Sencadas, V.; Zhang, J.; Zhang, L.; Konstantinov, K.; Guo, Z.; Liu, H. K. An All-Integrated Anode via Interlinked Chemical Bonding between Double-Shelled-Yolk-Structured Silicon and Binder for Lithium-Ion Batteries. Adv. Mater. 2017, 29, No. 1703028.

(6) Yang, J.; Wang, Y.; Li, W.; et al. Amorphous $\mathrm{TiO}_{2}$ Shells: A Vital Elastic Buffering Layer on Silicon Nanoparticles for High-Performance and Safe Lithium Storage. Adv. Mater. 2017, 29, No. 1700523.

(7) Luo, W.; Wang, Y.; Wang, L.; et al. Silicon/mesoporous carbon/ crystalline $\mathrm{TiO}_{2}$ nanoparticles for highly stable lithium storage. ACS Nano 2016, 10, 10524-10532.

(8) Luo, W.; Chen, X.; Xia, Y.; et al. Surface and Interface Engineering of Silicon-Based Anode Materials for Lithium-Ion Batteries. Adv. Energy Mater. 2017, 7, No. 1701083.

(9) Yang, F.; Gao, H.; Chen, J.; Guo, Z. Phosphorus-Based Materials as the Anode for Sodium-Ion Batteries. Small Methods 2017, 1, No. 1700216.

(10) Zhao, F.; Han, N.; Huang, W.; Li, J.; Ye, H.; Chen, F.; Li, Y. Nanostructured $\mathrm{CuP}_{2} / \mathrm{C}$ Composites as High-Performance Anode Materials for Sodium Ion Batteries. J. Mater. Chem. A 2015, 3, 2175421759.

(11) Wang, W.; Li, J.; Bi, M.; Zhao, Y.; Chen, M.; Fang, Z. Dual Function Flower-like CoP/C Nanosheets: High Stability Lithium-Ion Anode and Excellent Hydrogen Evolution Reaction Catalyst. Electrochim. Acta 2018, 259, 822-829.

(12) Lu, Y.; Zhou, P.; Lei, K.; Zhao, Q.; Tao, Z.; Chen, J. Selenium Phosphide $\left(\mathrm{Se}_{4} \mathrm{P}_{4}\right)$ as a New and Promising Anode Material for Sodium-Ion Batteries. Adv. Energy Mater. 2017, 7, No. 1601973.

(13) Von Lim, Y.; Huang, S.; Zhang, Y.; Kong, D.; Wang, Y.; Guo, L.; Zhang, J.; Shi, Y.; Chen, T. P.; Ang, L. K.; Yang, H. Bifunctional Porous Iron Phosphide/Carbon Nanostructure Enabled High-Performance Sodium-Ion Battery and Hydrogen Evolution Reaction. Energy Storage Mater. 2018, 15, 98-107.

(14) Wang, X.; Na, Z.; Yin, D.; Wang, C.; Huang, G.; Wang, L. Nanosized FexNi 2 -XP Embedded Phosphorus-Doped Carbon Nanorods with Superior Lithium Storage Performance. Energy Storage Mater. 2018, 12, 103-109.

(15) Jiang, J.; Wang, C.; Li, W.; Yang, Q. One-Pot Synthesis of Carbon-Coated $\mathrm{Ni}_{5} \mathrm{P}_{4}$ Nanoparticles and CoP Nanorods for High-Rate 
and High-Stability Lithium-Ion Batteries. J. Mater. Chem. A 2015,3, $23345-23351$.

(16) Jiang, J.; Zhu, K.; Fang, Y.; Wang, H.; Ye, K.; Yan, J.; Wang, G.; Cheng, K.; Zhou, L.; Cao, D. Coralloidal Carbon-Encapsulated CoP Nanoparticles Generated on Biomass Carbon as a High-Rate and Stable Electrode Material for Lithium-Ion Batteries. J. Colloid Interface Sci. 2018, 530, 579-585.

(17) Fan, X.; Mao, J.; Zhu, Y.; Luo, C.; Suo, L.; Gao, T.; Han, F.; Liou, S.-C.; Wang, C. Superior Stable Self-Healing $\mathrm{SnP}_{3}$ Anode for SodiumIon Batteries. Adv. Energy Mater. 2015, 5, No. 1500174.

(18) Zhang, C.; Jiao, G.; Kong, F.; Wang, J.; Tao, S.; Zhang, L.; Qian, B.; Chao, Y. Hierarchical $\mathrm{Co}_{2} \mathrm{P}$ Microspheres Assembled from Nanorods Grown on Reduced Graphene Oxide as Anode Material for Lithium-Ion Batteries. Appl. Surf. Sci. 2018, 459, 665-671.

(19) Liu, Q.; Luo, Y.; Chen, W.; Yan, Y.; Xue, L.; Zhang, W.CoP 3 PPy Microcubes as Anode for Lithium-Ion Batteries with Improved Cycling and Rate Performance. Chem. Eng. J. 2018, 347, 455-461.

(20) Zhu, J.; Wu, Q.; Key, J.; Wu, M.; Shen, P. K. Self-Assembled Superstructure of Carbon-Wrapped, Single-Crystalline $\mathrm{Cu}_{3} \mathrm{P}$ Porous Nanosheets: One-Step Synthesis and Enhanced Li-Ion Battery Anode Performance. Energy Storage Mater. 2018, 15, 75-81.

(21) Han, Z.; Wang, B.; Liu, X.; Wang, G.; Wang, H.; Bai, J. Peapodlike One-Dimensional (1D) CoP Hollow Nanorods Embedded into Graphene Networks as an Anode Material for Lithium-Ion Batteries. J. Mater. Sci. 2018, 53, 8445-8459.

(22) Lu, A.; Zhang, X.; Chen, Y.; Xie, Q.; Qi, Q.; Ma, Y.; Peng, D.-L. Synthesis of $\mathrm{CO}_{2} \mathrm{P} /$ Graphene Nanocomposites and Their Enhanced Properties as Anode Materials for Lithium Ion Batteries. J. Power Sources 2015, 295, 329-335.

(23) Xu, X.; Liu, J.; Hu, R.; Liu, J.; Ouyang, L.; Zhu, M. Self-Supported CoP Nanorod Arrays Grafted on Stainless Steel as an Advanced Integrated Anode for Stable and Long-Life Lithium-Ion Batteries. Chem. - Eur. J. 2017, 23, 5198-5204.

(24) Yang, D.; Zhu, J.; Rui, X.; Tan, H.; Cai, R.; Hoster, H. E.; Yu, D. Y. W.; Hng, H. H.; Yan, Q. Synthesis of Cobalt Phosphides and Their Application as Anodes for Lithium Ion Batteries. ACS Appl. Mater. Interfaces 2013, 5, 1093-1099.

(25) Tong, Y. X.; Li, X. M.; Xie, L. J.; Su, F. Y.; Li, J. P.; Sun, G. H.; Gao, Y. D.; Zhang, N.; Wei, Q.; Chen, C. M. Nitrogen-Doped Hierarchical Porous Carbon Derived from Block Copolymer for Supercapacitor. Energy Storage Mater. 2016, 3, 140-148.

(26) Chhowalla, M.; Shin, H. S.; Eda, G.; Li, L.-J.; Loh, K. P.; Zhang, H. The Chemistry of Two-Dimensional Layered Transition Metal Dichalcogenide Nanosheets. Nat. Chem. 2013, 5, 263-275.

(27) Stern, L.-A.; Feng, L.; Song, F.; Hu, X. Ni $i_{2} \mathrm{P}$ as a Janus Catalyst for Water Splitting: The Oxygen Evolution Activity of $\mathrm{Ni}_{2} \mathrm{P}$ Nanoparticles. Energy Environ. Sci. 2015, 8, 2347-2351.

(28) Wang, X.; Kolen'ko, Y. V.; Liu, L. Direct Solvothermal Phosphorization of Nickel Foam to Fabricate Integrated $\mathrm{Ni}_{2} \mathrm{P}$ Nanorods/Ni Electrodes for Efficient Electrocatalytic Hydrogen Evolution. Chem. Commun. 2015, 51, 6738-6741.

(29) Xiao, P.; Sk, M. A.; Thia, L.; Ge, X.; Lim, R. J.; Wang, J.-Y.; Lim, K. H.; Wang, X. Molybdenum Phosphide as an Efficient Electrocatalyst for the Hydrogen Evolution Reaction. Energy Environ. Sci. 2014, 7, 2624-2629.

(30) Yin, Y.; Rioux, R. M.; Erdonmez, C. K.; Hughes, S.; Somorjai, G. A.; Ivisatos, A. P. Formation of Hollow Nanocrystals Through the Nanoscale Kirkendall Effect. Science 2004, 304, 711-714.

(31) Hu, H.; Guan, B.; Xia, B.; Lou, X. W. D. Designed Formation of $\mathrm{CO}_{3} \mathrm{O}_{4} / \mathrm{NiCO}_{2} \mathrm{O}_{4}$ Double-Shelled Nanocages with Enhanced Pseudocapacitive and Electrocatalytic Properties. J. Am. Chem. Soc. 2015, 137, 5590-5595.

(32) Liu, J.; Xu, X.; Hu, R.; Yang, L.; Zhu, M. Uniform Hierarchical $\mathrm{Fe}_{3} \mathrm{O}_{4} @$ Polypyrrole Nanocages for Superior Lithium Ion Battery Anodes. Adv. Energy Mater. 2016, 6, No. 1600256.

(33) Xiao, Y.; Sun, P.; Cao, M. Core-Shell Bimetallic Carbide Nanoparticles Confined in a Three-Dimensional N-Doped Carbon Conductive Network for Efficient Lithium Storage. ACS Nano 2014, 8, $7846-7857$.
(34) Wei, W.; Yang, S.; Zhou, H.; Lieberwirth, I.; Feng, X.; Mullen, K. 3D Graphene Foams Cross-Linked with Pre-Encapsulated $\mathrm{Fe}_{3} \mathrm{O}_{4}$ Nanospheres for Enhanced Lithium Storage. Adv. Mater. 2013, 25, 2909-2914.

(35) Worsley, M. A.; Pauzauskie, P. J.; Olson, T. Y.; Biener, J.; Satcher, J. H.; Baumann, T. F. Synthesis of Graphene Aerogel with High Electrical Conductivity. J. Am. Chem. Soc. 2010, 132, 14067-14069.

(36) Cabana, J.; Monconduit, L.; Larcher, D.; Palacín, M. R. Beyond Intercalation-Based Li-Ion Batteries: The State of the Art and Challenges of Electrode Materials Reacting Through Conversion Reactions. Adv. Mater. 2010, 22, E170-E192.

(37) Palacín, M. R. Recent Advances in Rechargeable Battery Materials: A Chemist's Perspective. Chem. Soc. Rev. 2009, 38, 25652575.

(38) Yu, X. Y.; Yu, L.; Wu, H. B.; Lou, X. W. Formation of Nickel Sulfide Nanoframes from Metal-Organic Frameworks with Enhanced Pseudocapacitive and Electrocatalytic Properties. Angew. Chem., Int. Ed. 2015, 54, 5331- 5335 .

(39) Ren, W.; Qin, M.; Zhu, Z.; Yan, M.; Li, Q.; Zhang, L.; Liu, D.; Mai, L. Activation of Sodium Storage Sites in Prussian Blue Analogues via Surface Etching. Nano Lett. 2017, 17, 4713-4718.

(40) Li, Z.; Zhang, L.; Ge, X.; Li, C.; Dong, S.; Wang, C.; Yin, L. CoreShell Structured $\mathrm{CoP} / \mathrm{FeP}$ Porous Microcubes Interconnected by Reduced Graphene Oxide as High Performance Anodes for Sodium Ion Batteries. Nano Energy 2017, 32, 494-502.

(41) Gao, H.; Zhou, T.; Zheng, Y.; Liu, Y.; Chen, J.; Liu, H.; Guo, Z. Integrated Carbon/Red Phosphorus/Graphene Aerogel 3D Architecture via Advanced Vapor-Redistribution for High-Energy Sodium-Ion Batteries. Adv. Energy Mater. 2016, 6, No.1601037.

(42) Li, H.; Yang, P.; Chu, D.; Li, H. Selective Maltose Hydrogenation to Maltitol on a Ternary $\mathrm{Co}-\mathrm{P}-\mathrm{B}$ Amorphous Catalyst and the Synergistic Effects of Alloying B and P. Appl. Catal., A 2007, 325, 3440.

(43) Grosvenor, A. P.; Wik, S. D.; Cavell, R. G.; Mar, A. Examination of the Bonding in Binary Transition-Metal Monophosphides MP $(\mathrm{M}=$ $\mathrm{Cr}, \mathrm{Mn}, \mathrm{Fe}, \mathrm{Co}$ ) by X-Ray Photoelectron Spectroscopy. Inorg. Chem. 2005, 44, 8988-8998.

(44) Gao, H.; Zhou, T.; Zheng, Y.; Zhang, Q.; Liu, Y.; Chen, J.; Liu, H.; Guo, Z. CoS Quantum Dot Nanoclusters for High-Energy Potassium-Ion Batteries. Adv. Funct. Mater. 2017, 27, No. 1702634.

(45) Yang, J.; Zhang, Y.; Sun, C.; Liu, H.; Li, L.; Si, W.; Huang, W.; Yan, Q.; Dong, X. Graphene and Cobalt Phosphide Nanowire Composite as an Anode Material for High Performance Lithium-Ion Batteries. Nano Res. 2016, 9, 612-621.

(46) Lu, Y.; Tu, J. P.; Xiang, J. Y.; Wang, X. L.; Zhang, J.; Mai, Y. J.; Mao, S. X. Improved Electrochemical Performance of Self-Assembled Hierarchical Nanostructured Nickel Phosphide as a Negative Electrode for Lithium Ion Batteries. J. Phys. Chem. C 2011, 115, 23760-23767.

(47) Lu, Y.; Tu, J.; Xiong, Q.; Qiao, Y.; Wang, X.; Gu, C.; Mao, S. X. Synthesis of Dinickel Phosphide $\left(\mathrm{Ni}_{2} \mathrm{P}\right)$ for Fast Lithium-Ion Transportation: A New Class of Nanowires with Exceptionally Improved Electrochemical Performance as a Negative Electrode. RSC Adv. 2012, 2, 3430-3436.

(48) Carenco, S.; Surcin, C.; Morcrette, M.; Larcher, D.; Meźailles, N.; Boissiere, C.; Sanchez, C. Improving the Li-Electrochemical Properties of Monodisperse $\mathrm{Ni}_{2} \mathrm{P}$ Nanoparticles by Self-Generated Carbon Coating. Chem. Mater. 2012, 24, 688-697.

(49) Boyanov, S.; Zitoun, D.; Mérier, M.; Jumas, J. C.; Womes, M.; Monconduit, L. Comparison of the Electrochemical Lithiation/ Delitiation Mechanisms of FeP $(x=1,2,4)$ Based Electrodes in LiIon Batteries. J. Phys. Chem. C 2009, 113,21441-21452. 